\title{
HOMOMORPHISM REDUCTIONS ON POLISH GROUPS
}

\author{
KONSTANTINOS A. BEROS
}

\begin{abstract}
In an earlier paper, we introduced the following pre-order on the subgroups of a given Polish group: if $G$ is a Polish group and $H, L \subseteq G$ are subgroups, we say $H$ is homomorphism reducible to $L$ iff there is a continuous group homomorphism $\varphi: G \rightarrow G$ such that $H=\varphi^{-1}(L)$. We previously showed that there is a $K_{\sigma}$ subgroup, $L$, of the countable power of any locally compact Polish group, $G$, such that every $K_{\sigma}$ subgroup of $G^{\omega}$ is homomorphism reducible to $L$. In the present work, we show that this fails in the countable power of the group of increasing homeomorphisms of the unit interval.
\end{abstract}

\section{INTRODUCTION}

In the study of definable equivalence relations, the equivalence relations arising as coset equivalence relations on Polish groups have played a key role. For instance, the equivalence relation $E_{0}$ on $2^{\omega}$, given by

$$
x E_{0} y \Longleftrightarrow x(n)=y(n) \text {, for all but finitely many } n
$$

may be regarded as the coset equivalence relation of the subgroup

$$
\text { Fin }=\left\{x \in 2^{\omega}: x(n)=0 \text {, for all but finitely many } n\right\} \text {. }
$$

of the Polish group $2^{\omega}$, where the group operation is bitwise addition mod 2. Indeed, many important equivalence relations arise in this way, e.g., $E_{1}, E_{2}, E_{3}$, and $\ell^{\infty}$.

The most important means of comparing equivalence relations is the quasiorder of Borel reducibility. That is, if $E$ and $F$ are equivalence relations on Polish spaces $X$ and $Y$, respectively, one says that $E$ is Borel reducible to $F$ iff there is a Borel map $\varphi: X \rightarrow Y$ such that, for all $x, y \in X$,

$$
x E y \Longleftrightarrow \varphi(x) F \varphi(y) .
$$

The map $\varphi$ is called a Borel reduction of $E$ to $F$. The present work and our earlier paper [1] are motivated by the observation that, in many cases, Borel reductions between coset equivalence relations can be witnessed by continuous group homomorphisms between appropriate groups. For instance, let

$$
H_{1}=\left\{y \in 2^{\omega \times \omega}:\left(\forall^{\infty} m\right)(\forall m)(y(m, n)=0)\right\},
$$

and let $E_{1}$ be the coset relation of $H_{1}$. It is well-known that $E_{0}$ is Borel reducible to $E_{1}$. A Borel reduction witnessing this is $\varphi: 2^{\omega} \rightarrow 2^{\omega \times \omega}$, given by

$$
\varphi(x)(m, n)=\varphi(x)(m),
$$

2010 Mathematics Subject Classification. 03E15, 54H11, 22A05. 57S05, 
for each $x \in \mathbb{Z}_{2}^{\omega}$ and $m, n \in \omega$. Note that, in fact, Fin $=\varphi^{-1}\left[H_{1}\right]$, i.e., $\varphi$ is a continuous reduction of the subgroup Fin to $H_{1}$. Furthermore, the map $\varphi$ induces an injective homomorphism

$$
\tilde{\varphi}: 2^{\omega} / \text { Fin } \rightarrow 2^{\omega \times \omega} / H_{1}
$$

such that the diagram

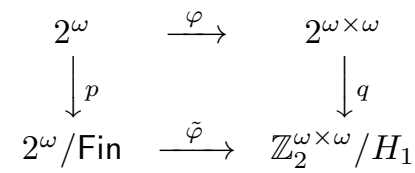

commutes. Here $p$ and $q$ denote the appropriate factor maps.

Abstracting from such specific examples leads to the following definition, already introduced in our paper [1].

Definition 1.1. Let $G$ be a Polish group. For subgroups $H$ and $L$ of $G$, we say that $H$ is group homomorphism reducible to $L$ iff there is a continuous group homomorphism $\varphi: G \rightarrow G$ such that $H=\varphi^{-1}[L]$. As a shorthand, we write $H \leq_{\mathrm{g}} L$.

As an alternative formulation, observe that $H \leq_{\mathrm{g}} L$ iff there is an embedding of $G / H$ into $G / L$ with a lifting which is a continuous endomorphism of $G$. Viewed in this light, our definition is not without precedent. For instance, Farah [2] considers maps between factors of Boolean algebras with liftings which are algebra homomorphisms.

The $K_{\sigma}$ equivalence relations play an important role in the general theory of equivalence relations, e.g., $E_{0}, E_{1}$ and $\ell^{\infty}$ are all $K_{\sigma}$. (Recall that a relation is $K_{\sigma}$ if it is the countable union of compact sets). In fact, much study has been done of such relations. See for instance [4]. In light of this, we directed much of our attention to the $K_{\sigma}$ subgroups of Polish groups in [1. We showed that there are $\leq_{\mathrm{g}}$-maximal $K_{\sigma}$ subgroups of $G^{\omega}$. We referred to such $\leq_{\mathrm{g}}$-maximal $K_{\sigma}$ subgroups as universal $K_{\sigma}$ subgroups of $G^{\omega}$. The equivalence relation analog is that of a Borelcomplete $K_{\sigma}$ equivalence relation. Though the requirement of universality is much stronger. For instance, $\ell^{\infty}$ is a Borel-complete $K_{\sigma}$ equivalence relation, but it is not universal in our sense.

As a counterpoint, we showed that there are no universal $K_{\sigma}$ subgroups of $S_{\infty}$. That is, for any $K_{\sigma}$ subgroup, $H$, of $S_{\infty}$, there is a $K_{\sigma}$ subgroup, $L \subseteq S_{\infty}$, such that $L \neq \varphi^{-1}[H]$, for every continuous homomorphism $\varphi: S_{\infty} \rightarrow S_{\infty}$. Since $S_{\infty}$ continuously embeds its own countable power, it followed immediately that $S_{\infty}^{\omega}$ has no universal $K_{\sigma}$ subgroup.

The cases in which universal $K_{\sigma}$ subgroups do not exist are interesting in that they indicate a degree of rigidity of the group in question. That is, if $G$ does not have a universal $K_{\sigma}$ subgroup, there is, in some sense, a shortage of continuous endomorphisms of $G$. It is worth remarking upon the fact that $S_{\infty}$ does have $\leq_{\mathrm{g}}$-maximal analytic subgroups. Thus, the rigidity which precludes universal $K_{\sigma}$ subgroups of $S_{\infty}$ is not sufficient to rule out universal analytic subgroups.

In the vein of our earlier results, we prove here an analogous theorem for the transformation group, $H_{+}([0,1])$, of increasing homeomporphisms of the unit interval.

Theorem 1.2. There is no universal $K_{\sigma}$ subgroup of $H_{+}([0,1])$. 
The proof of this result is in Section 3. As an aside, it follows from our earlier paper [1, Theorem 1.7] that there is an $F_{\sigma}$ subgroup of $H_{+}([0,1])$ of which every $K_{\sigma}$ subgroup is a continuous homomorphic pre-image. By Theorem 1.2. such a "universal $F_{\sigma}$ subgroup for $K_{\sigma}$ " cannot be $K_{\sigma}$ itself.

A direct corollary of Theorem 1.2 is

Corollary 1.3. There is no universal $K_{\sigma}$ subgroup of $H_{+}([0,1])^{\mathbb{N}}$.

Proof. Assume Theorem 1.2 holds. Observe that $H_{+}([0,1])^{\mathbb{N}}$ is isomorphic to the closed subgroup

$$
C=\left\{f \in H_{+}([0,1]):(\forall n)(f(1 / n)=1 / n)\right\}
$$

of $H_{+}([0,1])$. Let $\varphi: H_{+}([0,1])^{\mathbb{N}} \rightarrow C$ be an isomorphism and let $\psi$ be an isomorphism between $H_{+}([0,1])$ and a closed subgroup of $H_{+}([0,1])^{\mathbb{N}}$. Suppose, towards a contradiction, that $H_{+}([0,1])^{\mathbb{N}}$ has a universal $K_{\sigma}$ subgroup $H$. Let $\tilde{H}$ be the $K_{\sigma}$ sugbroup $\varphi(H)$ of $H_{+}([0,1])$. Fix an arbitrary $K_{\sigma}$ subgroup $L$ of $H_{+}([0,1])$. Note that $\psi(L)$ is a $K_{\sigma}$ subgroup of $H_{+}([0,1])^{\mathbb{N}}$ and hence, by the universality of $H$, there is a continuous endomorphism $\rho$ of $H_{+}([0,1])^{\mathbb{N}}$ such that $\psi(L)=\rho^{-1}(H)$. This implies that $L=(\varphi \circ \rho \circ \psi)^{-1}(H)$. As $L$ was arbitrary, this shows that $\varphi(H)$ is a universal $K_{\sigma}$ subgroup of $H_{+}([0,1])$, contradicting Theorem 1.2

In our study of $K_{\sigma}$ subgroups from 1, we also considered situations in which every $K_{\sigma}$ subgroup of a given Polish group is compactly generated. This is true in $\mathbb{Z}^{\omega}$, fo instance. In the final section of this paper, we expand upon these ideas and describe a large class of groups in which every $K_{\sigma}$ is group homomorphism reducible to a compactly generated subgroup, i.e., the compactly generated subgroups are $\leq_{\mathrm{g}}$-cofinal in the $K_{\sigma}$ subgroups.

\section{PReliminaries And notation}

The group $H_{+}([0,1])$ of increasing homeomorphisms of $[0,1]$ is a Polish group when equipped with the topology of uniform convergence, i.e., the relative topology inherited from $C([0,1])$. It is a peculiarity of $H_{+}([0,1])$ that this topology coincides with the topology of pointwise convergence. In the present setting, the latter is much easier to manipulate. The basic open sets in $H_{+}([0,1])$ are thus of the form

$$
U=\left\{f \in H_{+}([0,1]):(\forall i \leq k)\left(f\left(r_{i}\right) \in I_{i}\right)\right\},
$$

where $r_{0}, \ldots, r_{k} \in[0,1]$ and $I_{0}, \ldots, I_{k} \subseteq[0,1]$ are open intervals.

For $f \in H_{+}([0,1])$, let $\operatorname{supp}(f)=\{x \in[0,1]: f(x) \neq x\}$ be the support of $f$.

Let $\mathcal{N}$ denote the Baire space, i.e., $\mathbb{N}^{\mathbb{N}}$ with the product topology. For $\alpha \in \mathcal{N}$ and a finite sequence $s$ of natural numbers, $\alpha \supset s$ indicates that $s$ is an initial segment of $\alpha$.

Definition 2.1. Given $f \in H_{+}([0,1])$ and $\alpha \in \mathcal{N}$, define $f$ to be $\alpha$-continuous, iff, for each $k \in \mathbb{N}$ with $k \geq 1$, if $x, y \in[0,1]$ are such that $|x-y|<1 / \alpha(k)$, then $|f(x)-f(y)|<1 / k$.

Recall that a family $F \subseteq H_{+}([0,1])$ is equicontinuous iff there is an $\alpha \in \mathcal{N}$ such that each $f \in F$ is $\alpha$-continuous. For each $\alpha \in \mathcal{N}$, let

$$
K_{\alpha}=\left\{f \in H_{+}([0,1]): f, f^{-1} \text { are } \alpha \text {-continuous }\right\}
$$

and note that, by definition, each $K_{\alpha}$ is equicontinuous. 
Lemma 2.2. Suppose $f_{0}, f_{1}, \ldots \in H_{+}([0,1])$ and each $f_{n}$ is $\alpha$-continuous. If $f_{n} \rightarrow$ $f$ pointwise, then $f$ is also $\alpha$-continuous.

Proof. Fix $k \in \mathbb{N}$ and $x, y \in[0,1]$ such that $|x-y|<1 / \alpha(k)$. Assume that $k, \alpha(k)>1$, as otherwise the desired conclusion is automatic. Assume that $x<y$ and, since $1 / \alpha(k) \leq 1 / 2$, we may assume that $x \neq 0$ or $y \neq 1$. These two cases are analogous, so assume $y \neq 1$. Let $y^{\prime}>y$ be such that $\left|x-y^{\prime}\right|<1 / \alpha(k)$. For each $n$, $\left|f_{n}(x)-f_{n}\left(y^{\prime}\right)\right|<1 / k$ and hence

$$
\left|f(x)-f\left(y^{\prime}\right)\right|=\lim _{n}\left|f_{n}(x)-f_{n}\left(y^{\prime}\right)\right| \leq 1 / k .
$$

Since $f$ is a strictly increasing function,

$$
|f(x)-f(y)|<\left|f(x)-f\left(y^{\prime}\right)\right| \leq 1 / k .
$$

This completes the proof.

Each $K_{\alpha}$ is thus closed and, since the $K_{\alpha}$ are also equicontinuous, it follows from the Arzelà-Ascoli Theorem (see [5, §7.10]) that each $K_{\alpha}$ is compact. Conversely,

Lemma 2.3. If $K \subseteq H_{+}([0,1])$ is compact, then there exists $\alpha \in \mathcal{N}$ such that each $f \in K$ is $\alpha$-continuous.

Proof. Fix $k \in \mathbb{N}$, it suffices to determine a value for $\alpha(k)$ which is sufficiently large that $|f(x)-f(y)|<1 / k$, whenever $f \in K$ and $|x-y|<1 / \alpha(k)$. Indeed, fix open intervals $I_{0}, \ldots, I_{n} \subseteq(0,1)$ which cover $(0,1)$ and such that, if each $I_{i}=\left(a_{i}, b_{i}\right)$, then

$$
a_{i}<a_{i+1}<b_{i},
$$

for each $i \leq n-1$. Moreover, choose the $I_{i}$ such that $\left|b_{i+2}-a_{i}\right|<1 / k$, for each $i \leq n-2$. For each increasing tuple $r=\langle r(0), r(1), \ldots, r(n)\rangle \in(0,1)^{n+1}$, define the open set

$$
U_{r}=\left\{f \in H_{+}([0,1]):(\forall i \leq n)\left(f(r(i)) \in I_{i}\right\}\right.
$$

and observe that the $U_{r}$ cover $H_{+}([0,1])$. Since $K$ is compact, there exist $r_{0}<r_{1}<$ $\ldots<r_{p}$ such that $U_{r_{0}}, \ldots, U_{r_{p}}$ cover $K$. Let

$$
\varepsilon_{k}=\min \left(\left\{\left|r_{j}(i)-r_{j}(i+1)\right|: i<n \& j \leq p\right\}\right)
$$

and note that, for each $f \in K$ and $x, y \in[0,1]$, if $|x-y|<\varepsilon_{k}$, then $|f(x)-f(y)|<$ $1 / k$. Choose $\alpha(k) \geq 1 / \varepsilon_{k}$. This completes the proof.

If $K \subseteq H_{+}([0,1])$ is compact, then so is $\left\{f^{-1}: f \in K\right\}$. It thus follows from the last lemma that $K \subseteq K_{\alpha}$, for some $\alpha \in \mathcal{N}$.

In the interest of clarity, if $f, g$ are functions, $f g$ througout denotes the composite function $f \circ g$ and $f^{n}$ dentotes the $n$-fold iterate of $f$ (for $n \in \mathbb{N}$ ). Regarding elements of $\mathcal{N}$ as functions on $\mathbb{N}$, this notation applies equally to members of the Baire space. Similarly, if $f \in H_{+}([0,1])$ and $\varphi: H_{+}([0,1]) \rightarrow H_{+}([0,1])$ is an endomorphism, $\varphi f$ denotes the image of $f$ under $\varphi$, i.e., $\varphi(f)$.

The following facts are direct consequences of the definitions of $\alpha$-continuity and the $K_{\alpha}$.

(1) If $f$ is $\alpha$-continuous and $g$ is $\beta$-continuous, then $f g$ is $\beta \alpha$-continuous.

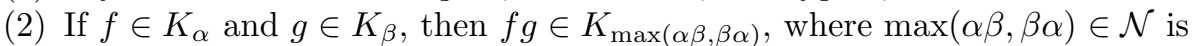
such that $\max (\alpha \beta, \beta \alpha)(k)=\max (\alpha \beta(k), \beta \alpha(k))$, for each $k \in \mathbb{N}$.

(3) If $f \in K_{\alpha}$ and $|x-y| \geq 1 / k$, for some $k$, then $|f(x)-f(y)| \geq 1 / \alpha(k)$.

Finally, for $A \subseteq H_{+}([0,1])$, let $\langle A\rangle$ designate the subgroup generated by $A$. 


\section{Proof of Theorem 1.2}

Lemma 3.1. There is an increasing homeomorphism $\sigma:[0,1] \rightarrow[0,1]$ such that $\sigma$ is supported on $[1 / 2,3 / 4]$ and the conjugacy class of $\sigma$ is dense.

Proof. Glasner-Weiss $\left[3\right.$ have shown that $H_{+}([0,1])$ contains a dense conjugacy class. Therefore, fix $\sigma \in H_{+}([0,1])$ such that the conjugacy class of $\sigma$ is dense. The objective of the proof is to modify $\sigma$ and produce $\tau$ which is supported on $[1 / 2,3 / 4]$ and still has dense conjugacy class.

Let $h:[0,1] \rightarrow[1 / 2,3 / 4]$ be a linear bijection. Define $\tau \in H_{+}([0,1])$ by

$$
\tau(x)= \begin{cases}h \sigma h^{-1}(x) & \text { if } x \in[1 / 2,3 / 4] \\ x & \text { otherwise. }\end{cases}
$$

It remains to show that the conjugacy class of $\tau$ is dense in $H_{+}([0,1])$. To this end, fix a basic open set $U \subseteq H_{+}([0,1])$ with $0<r_{0}<\ldots<r_{k}<1$ and nonempty open intervals $I_{0}, \ldots, I_{k} \subseteq(0,1)$ such that

$$
U=\left\{f \in H_{+}([0,1]):(\forall i \leq k)\left(f\left(r_{i}\right) \in I_{i}\right)\right\} .
$$

With no loss of generality, $\sup \left(I_{i}\right)<1$ and $\inf \left(I_{i}\right)>0$, for each $i \leq k$, as this only shrinks the open set $U$. Fix $a, b \in(0,1)$ such that $0<a<r_{0}, r_{k}<b<1$, $a<\min _{i \leq k}\left(\inf \left(I_{i}\right)\right)$ and $b>\max _{i \leq k}\left(\sup \left(I_{i}\right)\right)$. Let $g$ be the piecewise linear map defined such that the graph of $g$ has vertices $(0,0),(1 / 2, a),(3 / 4, b),(1,1)$. Note that $g \in H_{+}([0,1])$.

For each $i \leq k$, let $s_{i}=h^{-1} g^{-1}\left(r_{i}\right)$ and $J_{i}=h^{-1} g^{-1}\left(I_{i}\right)$. Since the conjugacy class of $\sigma$ is dense, there exists $f \in H_{+}([0,1])$ such that $f \sigma f^{-1}\left(s_{i}\right) \in J_{i}$, for each $i \leq k$. Define $f_{1} \in H_{+}([0,1])$ by

$$
f_{1}(x)= \begin{cases}h f h^{-1}(x) & \text { if } x \in[1 / 2,3 / 4] \\ x & \text { otherwise. }\end{cases}
$$

To complete the proof, it suffices to show that $g f_{1} \tau f_{1}^{-1} g^{-1} \in U$. Fix $i \leq k$ and observe that

$$
\begin{aligned}
g f_{1} \tau f_{1}^{-1} g^{-1}\left(r_{i}\right) & =g f_{1} \tau f_{1}^{-1} h\left(s_{i}\right) \\
& =g h f h^{-1} h \sigma h^{-1} h f^{-1} h^{-1} h\left(s_{i}\right) \\
& =g h f \sigma f^{-1}\left(s_{i}\right) \\
& \in g h\left(J_{i}\right) \\
& =I_{i} .
\end{aligned}
$$

This completes the proof.

Let $\sigma_{1}$ be as in the previous lemma, with dense conjugacy class and $\operatorname{supp}\left(\sigma_{1}\right) \subseteq$ $[1 / 2,3 / 4]$. Define $h \in H_{+}([0,1])$ such that the graph of $h$ is polygonal and the vertices of $h$ are the elements of the set

$$
\left\{\left(1-2^{-n}, 1-2^{-n-1}\right): n \in \mathbb{N}, n \geq 1\right\}
$$

of points in $[0,1]^{2}$, i.e., $h$ has vertices $(1 / 2,3 / 4),(3 / 4,7 / 8), \ldots$. To simplify notation, let $I_{n}$ denote the interval $\left[1-2^{-n}, 1-2^{-n-1}\right]$, for each $n \in \mathbb{N}$. In other words, $I_{0}=[0,1 / 2], I_{1}=[1 / 2,3 / 4]$, and so forth. For $n \geq 1$, define $\sigma_{n}=h^{n-1} \sigma_{1} h^{1-n}$ and observe that $\sigma_{n}$ is supported on the interval $I_{n}$. 
Given $\alpha \in \mathcal{N}$, define $L_{\alpha} \subseteq H_{+}([0,1])$ to be the set of those $f \in H_{+}([0,1])$ such that $f \uparrow I_{0}=$ id $\uparrow I_{0}$ and, for each $n \geq 1, f \uparrow I_{n}=\sigma_{n}^{i} \uparrow I_{n}$, for some $i \leq \alpha(n)$. If $f \in L_{\alpha}$ is the (unique) member of $L_{\alpha}$ such that $f \uparrow I_{n}=\sigma_{n}^{\delta(n)} \uparrow I_{n}$, for a given $\delta \leq \alpha$, then denote $f$ by $f_{\delta}$. Observe that $L_{\alpha}$ is a continuous image of the compact space

$$
X_{\alpha}=\prod_{n}\{0, \ldots, \alpha(n)\},
$$

via the map $\delta \mapsto f_{\delta}$. Each $L_{\alpha}$ is therefore compact.

In fact, the map $\delta \mapsto f_{\delta}$ is a homeomorphism between $X_{\alpha}$ and $L_{\alpha}$ with its subspace topology. It follows that the sets

$$
U_{s}=\left\{f_{\delta}: \delta \supset s\right\},
$$

with $s$ a finite sequence bounded by $\alpha$, form a basis for the relative topology on $L_{\alpha}$.

Given a $K_{\sigma}$ subgroup $H \subseteq H_{+}([0,1])$, the objective of the proof is to show that $H$ is not a universal subgroup. For this it is sufficient to show that there exists a compact set $L \subseteq H_{+}([0,1])$ such that, for every non-trivial continuous endomorphism $\varphi$ of $H_{+}([0,1])$, the image of $\langle L\rangle$ under $\varphi$ is not contained in $H$ and hence $\varphi$ is not a homomorphism reduction of $\langle L\rangle$ to $H$. Note that restriction to non-trivial endomorphisms of $H_{+}([0,1])$ is appropriate since, if $\langle L\rangle=\varphi^{-1}(H)$, then $\operatorname{ker}(\varphi) \subseteq\langle L\rangle$ and hence $\operatorname{ker}(\varphi) \neq H_{+}([0,1])$, as otherwise $H_{+}([0,1])=\langle L\rangle$, which is impossible because $H_{+}([0,1])$ is not itself $K_{\sigma}$.

To further simplify the argument, assume that $H$ is of the form $\bigcup_{m} K_{\beta_{m}}$, where $K_{\beta_{m}}$ is defined as in Section 2. It is possible to make this assumption as $H$ is contained in a set of the form $\bigcup_{m} K_{\beta_{m}}$. In addition, assume that $\left\{\beta_{m}: m \in \mathbb{N}\right\}$ is closed under composition and taking pointwise maxima, i.e., for each $m, n$ there exist $p, q$ such that $\beta_{m} \beta_{n}=\beta_{p}$ and $\max \left(\beta_{m}, \beta_{n}\right)=\beta_{q}$. The only purpose of these assumptions is to guarantee that, for any $m, n$, there exists $p$ such that if $f \in K_{\beta_{m}}$ and $g \in K_{\beta_{n}}$, then $f g, g f \in K_{\beta_{p}}$.

Observe that these simplifying assumptions introduce no loss of generality, since they only enlarge the set $\bigcup_{m} K_{\beta_{m}}$.

Henceforth, fix $\beta_{0}, \beta_{1}, \ldots \in \mathcal{N}$ as above. Choose $\alpha \in \mathcal{N}$ such that, for each $n \in \mathbb{N}$,

$$
\alpha(n)=\max \left(\left\{\beta_{i}^{j}(k): i, j, k \leq n\right\}\right)+1 .
$$

To complete the proof, it suffices to show that there is no continuous endomorphism of $H_{+}([0,1])$ which maps the compactly generated subgroup $\left\langle\{h\} \cup L_{\alpha}\right\rangle$ into $\bigcup_{m} K_{\beta_{m}}$. (Here $h$ is the map described above such that $\sigma_{n+1}=h \sigma_{n} h^{-1}$.) Indeed, suppose that, on the contrary, there exists an endomorphism $\varphi$ of $H_{+}([0,1])$ such that $\varphi$ maps $\left\langle\{h\} \cup L_{\alpha}\right\rangle$ into $\bigcup_{m} K_{\beta_{m}}$.

Lemma 3.2. There exists $m$ such that $\varphi\left(\{h\} \cup L_{\alpha}\right) \subseteq K_{\beta_{m}}$.

Proof. As a compact subset of a Polish space, $\varphi\left(L_{\alpha}\right)$ is Polish in its relative topology and thus, by the Baire Category Theorem, there exists an open set $V \subseteq H_{+}([0,1])$ and $m_{0} \in \mathbb{N}$ such that $\varphi\left(L_{\alpha}\right) \cap V$ is nonempty and $K_{\beta_{m_{0}}}$ is comeager on $\varphi\left(L_{\alpha}\right) \cap V$. Since $K_{\beta_{m_{0}}}$ is a closed set, $K_{\beta_{m_{0}}}$ must, in fact, contain $\varphi\left(L_{\alpha}\right) \cap V$. Let $U=\varphi^{-1}(V)$. It follows that $U \cap L_{\alpha}$ is nonempty and hence $\varphi\left(U_{s}\right) \subseteq K_{\beta_{m_{0}}}$, for some finite sequence $s$ which is bitwise bounded by $\alpha$. Observe that any $f \in L_{\alpha}$ is of the form

$$
\sigma_{1}^{i_{1}} \sigma_{2}^{i_{2}} \ldots \sigma_{|s|}^{i_{|s|}} g
$$


for some $g \in U_{s}$ and $i_{1}, \ldots, i_{|s|} \in \mathbb{Z}$, with each $\left|i_{p}\right| \leq 2 \alpha(p)$. Note that

$$
\sigma_{1}^{i_{1}}, \sigma_{2}^{i_{2}}, \ldots \sigma_{|s|}^{i_{|s|}} \in\left\langle L_{\alpha}\right\rangle
$$

for all such $i_{1}, \ldots, i_{|s|}$. Since $\varphi\left(\left\langle L_{\alpha}\right\rangle\right) \subseteq \bigcup_{m} K_{\beta_{m}}$, it now follows from the properties of $\beta_{0}, \beta_{1}, \ldots$ that there exists $m_{1} \in \mathbb{N}$ such that $K_{\beta_{m_{1}}}$ contains all elements of the form (3.1). In other words, $K_{\beta_{m_{1}}}$ contains $\varphi\left(L_{\alpha}\right)$.

Finally, since $\varphi h \in \bigcup_{m} K_{\beta_{m}}$, there exists $m_{2}$ with $\varphi h \in K_{\beta_{m_{2}}}$. It follows from the closure properties of the $\beta_{i}$ that $\varphi\left(\{h\} \cup L_{\alpha}\right) \subseteq K_{\beta_{m_{3}}}$, for some $m_{3} \in \mathbb{N}$. This proves the lemma.

Let $\beta_{m}$ be as in the previous lemma, with $\varphi\left(\{h\} \cup L_{\alpha}\right) \subseteq K_{\beta_{m}}$. For simplicity, write $\beta=\beta_{m}$.

Since the conjugacy class of $\sigma_{1}$ is dense, if $\varphi \sigma_{1}=\mathrm{id}$, then $\varphi f=\mathrm{id}$, for each $f \in$ $H_{+}([0,1])$. Indeed, if $\sigma_{1} \in \operatorname{ker}(\varphi)$, then $\operatorname{ker}(\varphi)$ contains the entire conjugacy class of $\sigma_{1}$, as it is a normal subgroup of $H_{+}([0,1])$. This implies that $\operatorname{ker}(\varphi)=H_{+}([0,1])$, since $\operatorname{ker}(\varphi)$ is also closed. On the other hand, this violates the assumption that $\varphi$ is non-trivial. Therefore, choose $x_{1} \in[0,1]$ and $k \in \mathbb{N}$ such that

$$
\left|x_{1}-\varphi \sigma_{1}\left(x_{1}\right)\right| \geq 1 / k \text {. }
$$

Define $x_{n+1}=\varphi h\left(x_{n}\right)$, for each $n \geq 1$, where $h$ is again as above. Observe that

$$
\begin{aligned}
\left|x_{2}-\varphi \sigma_{2}\left(x_{2}\right)\right| & =\left|\varphi h\left(x_{1}\right)-\varphi \sigma_{2} \varphi h\left(x_{1}\right)\right| \\
& =\left|\varphi h\left(x_{1}\right)-\varphi h \varphi \sigma_{1} \varphi h^{-1} \varphi h\left(x_{1}\right)\right| \\
& =\left|\varphi h\left(x_{1}\right)-\varphi h \varphi \sigma_{1}\left(x_{1}\right)\right| \\
& \geq 1 / \beta(k),
\end{aligned}
$$

since $\varphi h \in K_{\beta}$ and hence $\varphi h^{-1}$ is $\beta$-continuous. Iterating this argument, it follows that

$$
\left|x_{n}-\varphi \sigma_{n}\left(x_{n}\right)\right| \geq 1 / \beta^{n-1}(k)
$$

for each $n \geq 1$.

Let $n \geq k, m$ and let $p=\alpha(n)$. Recall that, by the choice of $\alpha$,

$$
p>\beta^{n}(k) \text {. }
$$

Consider the points $x_{n}, \varphi \sigma_{n}\left(x_{n}\right), \varphi \sigma_{n}^{2}\left(x_{n}\right), \ldots, \varphi \sigma_{n}^{p}\left(x_{n}\right)$, where $p \geq 1$. Because $\varphi \sigma_{n}$ is an increasing homeomorphism of $[0,1]$ and $\varphi \sigma_{n}\left(x_{n}\right) \neq x_{n}$, either

$$
x_{n}<\varphi \sigma_{n}\left(x_{n}\right)<\varphi \sigma_{n}^{2}\left(x_{n}\right)<\ldots<\varphi \sigma_{n}^{p}\left(x_{n}\right)
$$

or

$$
x_{n}>\varphi \sigma_{n}\left(x_{n}\right)>\varphi \sigma_{n}^{2}\left(x_{n}\right)>\ldots>\varphi \sigma_{n}^{p}\left(x_{n}\right) .
$$

In either case, there must exist $i<p$ such that

$$
\left|\varphi \sigma_{n}^{i}\left(x_{n}\right)-\varphi \sigma_{n}^{i+1}\left(x_{n}\right)\right| \leq 1 / p
$$

since the length of $[0,1]$ is 1 . Since $1 / p<1 / \beta^{n}(k)$, it follows that $\varphi \sigma_{n}^{-i}$ is not $\beta$-continuous, since

$$
\left|\varphi \sigma_{n}^{i}\left(x_{n}\right)-\varphi \sigma_{n}^{i+1}\left(x_{n}\right)\right| \leq 1 / p<1 / \beta^{n}(k),
$$

but

$$
\left|\varphi \sigma_{n}^{-i} \varphi \sigma_{n}^{i}\left(x_{n}\right)-\varphi \sigma_{n}^{-i} \varphi \sigma_{n}^{i+1}\left(x_{n}\right)\right|=\left|x_{n}-\varphi \sigma_{n}\left(x_{n}\right)\right| \nless 1 / \beta^{n-1}(k),
$$


by (3.2). Thus $\varphi \sigma_{n}^{i} \notin K_{\beta}$. This is a contradiction, since the choice of $\alpha$ implies that $\sigma_{n}^{i} \in L_{\alpha}$ and $\varphi$ maps $L_{\alpha}$ into $K_{\beta}$.

\section{Compactly Generated Subgroups}

We consider a criterion on a group $G$ which guarantees that the compactly generated subgroups of $G^{\omega}$ are $\leq_{\mathrm{g}}$-cofinal in the $K_{\sigma}$ subgroups. We begin with a couple of motivating examples.

Example 4.1. We showed in [1] that every $K_{\sigma}$ subgroup of $\mathbb{Z}^{\omega}$ is compactly generated. The argument was similar in character to the proof that $\mathbb{Z}$ is a principal ideal domain. For instance, the countable dense subgroup $\left\{x \in \mathbb{Z}^{\omega}:\left(\forall^{\infty} n\right)(x(n)=0)\right\}$ is generated by the compact set

$$
\left\{0^{n \frown 1} \overline{0}: n \in \omega\right\} \cup\{\overline{0}\} .
$$

For the sake of the next example, we say that $x$ is divisible in $H$ (where $H$ is a group), iff, for each $n$, there exists $y \in H$ such that $y^{n}=x$.

Example 4.2. Consider the Polish group $\mathbb{Q}^{\omega}$, where $\mathbb{Q}$ is equipped with the discrete topology. There are $K_{\sigma}$ subgroups, e.g.,

$$
H=\left\{x \in \mathbb{Q}^{\omega}:(\forall n)(n \geq 1 \Longrightarrow x(n)=0)\right\},
$$

which are $K_{\sigma}$, but not compactly generated. In fact, the subgroup $H$ is not $\leq_{\mathrm{g}^{-}}$ reducible to any compactly generated subgroup of $\mathbb{Q}^{\omega}$. To see this, suppose that $\varphi: \mathbb{Q}^{\omega} \rightarrow \mathbb{Q}^{\omega}$ is a group homomorphism with $H=\varphi^{-1}(\langle K\rangle)$, for some compact set $K \subseteq \mathbb{Q}^{\omega}$. Note that, since $H$ is a divisible subgroup of $\mathbb{Q}^{\omega}$, the subgroup $\langle K\rangle$ must contain elements which are divisible in $\langle K\rangle$. This, however, is impossible since it would imply that there is some $n \in \omega$ such that $\{x(n): x \in K\}$ is infinite.

The results in this section were motivated by the observation that the last example hinges on the fact that there are no compactly generated subgroups, $H$, of $\mathbb{Q}^{\omega}$ such that some element of $H$ is divisible in $H$. On the other hand, for instance, in the permutation group of the natural numbers, there are compactly generated subgroups which have divisible elements. Consider, for instance, the following.

Example 4.3. For each $n \geq 1$, let

$$
\pi_{n}=\prod_{i \in \omega}[2 n i, 2 n i+2, \ldots, 2 n(i+1), 2 n i+1,2 i n+3, \ldots, 2 n(i+1)+1]
$$

and observe that $\left(\pi_{n}\right)^{n}=\pi_{1}$, for each $n \geq 1$. It follows that $\pi_{1}$ is divisible in the subgroup generated by the $\pi_{n}$. If we let $H$ be the subgroup generated by the $\pi_{n}$, together with the finite support permutations, we obtain a compactly generated subgroup with a divisible element. Indeed, $H$ is compactly generated, since it is generated by the compact set

$$
\{[n, n+1]: n \in \omega\} \cup\left\{\pi_{n}^{\prime}: n \in \omega\right\} \cup\{\operatorname{id}\},
$$

where

$$
\pi_{n}^{\prime}=\prod_{i \geq n}[2 n i, 2 n i+2, \ldots, 2 n(i+1), 2 n i+1,2 i n+3, \ldots, 2 n(i+1)+1] .
$$

In turns out that, while there are non-compactly generated $K_{\sigma}$ subgroups of $S_{\infty}$, every $K_{\sigma}$ subgroup of $S_{\infty}$ is, in fact, $\leq_{\mathrm{g}}$-reducible to a compactly generated subgroup. This is a consequence of Theorem 4.5 below. 
Definition 4.4. A subgroup $H$ of a topological group $G$ is almost compactly generated iff there is a a compact set $K \subseteq G$ and a continuous, injective group homomorphism $\varphi: G \rightarrow G$ such that $H=\varphi^{-1}(\langle K\rangle)$.

The following theorem and remark together characterize those Polish groups of the form $G^{\omega}$ in which every $K_{\sigma}$ subgroup is almost compactly generated.

Theorem 4.5. Let $G$ be a Polish group. If $G$ has a dense subgroup which is a oneto-one continuous homomorphic pre-image of some compactly generated subgroup of $G^{\omega}$, then every $K_{\sigma}$ subgroup of $G^{\omega}$ is almost compactly generated.

Remark. Suppose that the hypothesis of Theorem 4.5 fails, i.e., there is no dense subgroup of $G$ which is an injective homomorphic pre-image of some compactly generated subgroup of $G^{\omega}$. One can define a $K_{\sigma}$ subgroup of $G^{\omega}$ which is not almost compactly generated by taking a dense $K_{\sigma}$ subgroup $D \subseteq G$ and letting

$$
\tilde{D}=\left\{\xi \in G^{\omega}:(\forall i)(\xi(i)=\xi(0) \in D)\right\} .
$$

It follows that $\tilde{D}$ is not almost compactly generated. Otherwise, there would exist an injective continuous endomorphism $\varphi: G^{\omega} \rightarrow G^{\omega}$ and a compact set $K \subseteq G^{\omega}$ such that $\tilde{D}=\varphi^{-1}(\langle K\rangle)$. Let $\psi: G \rightarrow G^{\omega}$ be given by $\psi(x)(i)=x$, for all $i$. Then, contrary to assumption, $D=(\varphi \circ \psi)^{-1}(\langle K\rangle)$.

Theorem 4.5 yields the following immediate corollary.

Corollary 4.6. If $G$ is a Polish group with a dense compactly generated subgroup, then every $K_{\sigma}$ subgroup of $G^{\omega}$ is almost compactly generated.

In general, we can consider other groups besides those of the form $G^{\omega}$.

Corollary 4.7. Suppose that $G$ is a Polish group such that $G^{\omega}$ isomorphic to a subgroup of $G$. If $G$ has a dense almost compactly generated subgroup, then every $K_{\sigma}$ subgroup of $G$ is almost compactly generated.

Proof. Let $\varphi_{1}: G \rightarrow G^{\omega}$ and $\varphi_{2}: G^{\omega} \rightarrow G$ be isomorphic embeddings. Fix a $K_{\sigma}$ subgroup $H \subseteq G$. By Theorem 4.5, there exists a compact set $K \subseteq G^{\omega}$ and a continuous injective homomorphism $\varphi: G^{\omega} \rightarrow G^{\omega}$ such that $\varphi_{1}(H)=\varphi^{-1}(\langle K\rangle)$. It now follows from the injectivity of $\varphi_{2}$ that

$$
H=\left(\varphi_{2} \circ \varphi \circ \varphi_{1}\right)^{-1}\left(\left\langle\varphi_{2}(K)\right\rangle\right) .
$$

In other words, $H$ is almost compactly generated.

Proof of Theorem 4.5. We start with a special case. Assume there is a dense subgroup $D \subseteq G$ and a continuous, injective homomorphism $\psi: G \rightarrow G$ such that $D=\psi^{-1}(\langle K\rangle)$, for some compact set $K \subseteq G$. We will show that every $K_{\sigma}$ subgroup of $G$ is a continuous homomorphic preimage of some compactly generated subgroup of $G^{\omega}$. We let $e$ denote the identity element of $G$.

To this end, fix a $K_{\sigma}$ subgroup $H=\bigcup_{n} H_{n} \subseteq G$, with each $H_{n}$ compact. For each $n$, let $F_{n} \subseteq D$ be a finite $\frac{1}{n}$-net for $H_{n}$. Let

$$
\tilde{H}_{n}=\bigcup_{y \in F_{n}} y^{-1} \cdot \operatorname{cl}\left(B_{\frac{1}{n}}(y) \cap H_{n}\right)
$$

and define $C_{n} \subseteq G^{\omega}$ to be the set of $\xi \in G^{\omega}$ such that

(1) $(\forall i<n)\left(\xi(i) \in \psi\left(\tilde{H}_{n}\right)\right)$ and

(2) $(\forall i \geq n)\left(\xi(i) \in \psi\left(H_{n}\right)\right)$. 
As a product of compact sets, each $C_{n}$ is a compact subset of $G^{\omega}$. In fact, $\bigcup_{n} C_{n}$ is itself compact. To see this, suppose that $\xi_{0}, \xi_{1}, \ldots \in \bigcup_{n} C_{n}$. In the first place, suppose that there is an $m$ such that infinitely many $\xi_{n} \in C_{m}$. In this case, there is a convergent subsequence of $\left(\xi_{n}\right)$, since each $C_{m}$ is compact. On the other hand, suppose that, for each $m$, there are only finitely many $n$ such that $\xi_{n} \in C_{m}$. Extract a subsequence $n_{0}<n_{1}<\ldots$ such that $\xi_{n_{p}} \in C_{p}$, for each $p \in \omega$. For each $p$, let $\eta_{p} \in G^{\omega}$ be such that

$$
\xi_{n_{p}}(i)=\psi\left(\eta_{p}(i)\right),
$$

for each $i \in \omega$. By the injectivity of $\psi$, we must have

$$
\eta_{p}(i) \in \tilde{H}_{n_{p}} \subseteq B_{\frac{1}{n_{p}}}(e),
$$

for each $p$ and $i<p$. It follows that $\eta_{p} \rightarrow \bar{e}$, as $p \rightarrow \infty$. Hence, $\xi_{n_{p}} \rightarrow \bar{e}$, since $\psi$ is a continuous homomorphism.

Now define a compact set $K^{*} \subseteq G^{\omega}$ by

$$
K^{*}=\left\{\xi \in K^{\omega}:\left(\exists^{\leq 1} i\right)(\xi(i) \neq e)\right\}
$$

and let $\varphi: G \rightarrow G^{\omega}$ be given by $\varphi(x)=\overline{\psi(x)}$. We check that

$$
H=\varphi^{-1}\left(\left\langle K^{*} \cup \bigcup_{n} C_{n}\right\rangle\right) .
$$

In the first place, suppose that $x \in H$, say $x \in H_{n}$. Let $y \in F_{n}$ and $z \in \tilde{H}_{n}$ be such that $y^{-1} x=z$. It follows that

$$
\begin{aligned}
\varphi(x) & =\overline{\psi(x)} \\
& =\underbrace{\left((\psi(y))^{n \frown \bar{e})}\right.}_{\in\left\langle K^{*}\right\rangle} \cdot \underbrace{\left((\psi(z))^{n \frown \overline{\psi(x)}}\right)}_{\in C_{n}} .
\end{aligned}
$$

Hence, $\varphi(x) \in\left\langle K^{*} \cup \bigcup_{n} C_{n}\right\rangle$.

On the other hand, suppose that $\varphi(x) \in\left\langle K^{*} \cup \bigcup_{n} C_{n}\right\rangle$. Let $n_{0} \in \omega$ and $w$ be a group word, with $\xi_{0}, \ldots \xi_{k} \in C_{n_{0}}$ and $\eta_{0} \ldots, \eta_{p} \in K^{*}$ such that

$$
\varphi(x)=w\left(\xi_{0}, \ldots, \xi_{k}, \eta_{0}, \ldots, \eta_{p}\right) .
$$

Let $i \geq n_{0}$ be large enough that

$$
\eta_{0}(i)=\ldots=\eta_{p}(i)=e .
$$

It follows that

$$
\begin{aligned}
\psi(x) & =\varphi(x)(i) \\
& =w\left(\xi_{0}(i), \ldots, \xi_{k}(i), \eta_{0}(i), \ldots, \eta_{p}(i)\right) \\
& =w\left(\xi_{0}(i), \ldots, \xi_{k}(i), e, \ldots, e\right) \in\left\langle\psi\left(H_{n_{0}}\right)\right\rangle .
\end{aligned}
$$

Since $\psi$ is injective, it follows that $x \in\left\langle H_{n_{0}}\right\rangle$.

We now turn to the general case in which there is a dense subgroup $D \subseteq G$, a compact set $K \subseteq G^{\omega}$, and an injective homomorphism $\psi: G \rightarrow g^{\omega}$ such that $H=\psi^{-1}(\langle K\rangle)$.

Consider the subgroup

$$
D^{*}=\left\{\xi \in D^{\omega}:\left(\forall^{\infty} i\right)(\xi(i)=e)\right\}
$$


and observe that $D^{*}$ is dense in $G^{\omega}$. Define $\psi^{*}: G^{\omega} \rightarrow\left(G^{\omega}\right)^{\omega}$ by

$$
\psi^{*}(\xi)(n, i)=\psi(x)(i)
$$

Now let

$$
K^{*}=\left\{\eta \in K^{\omega}:\left(\exists^{\leq 1} n\right)(\eta(n) \neq \bar{e})\right\} .
$$

Note that $K^{*}$ is compact and $D^{*}=\left(\psi^{*}\right)^{-1}\left(\left\langle K^{*}\right\rangle\right)$. We may therefore apply the special case above to the group $G^{\omega}$ and use the fact that $G^{\omega} \cong\left(G^{\omega}\right)^{\omega}$ to complete the proof.

\section{REFERENCES}

[1] Konstantinos A. Beros. Universal subgroups of Polish groups. Journal of Symbolic Logic, 79(4):1148-1183, 2014.

[2] Ilijas Farah. Completely additive liftings. Bulletin of Symbolic Logic, 4(1):37-54, March 1998.

[3] Eli Glasner and Benjamin Weiss. The topological Rohlin property and topological entropy. American Journal of Mathematics, 123(6):1055-1070, December 2001.

[4] Christian Rosendal. Cofinal families of Borel equivalence relations and quasiorders. Journal of Symbolic Logic, 70(4):1325-1340, December 2005.

[5] Halsey L. Royden. Real Analysis. Prentice-Hall, 3rd edition, 1988.

Department of Mathematics, University of North Texas, General Academics Building 435, 1155 Union Circle, \#311430, Denton, TX 76203-5017

E-mail address: beros@unt.edu 